University of Nebraska - Lincoln

DigitalCommons@University of Nebraska - Lincoln

$12-2009$

\title{
Expression artifact with retroviral vectors based on $\mathrm{pBMN}$
}

\author{
Yoshitaka Fukunaga \\ University of Nebraska Medical Center, Omaha \\ Robert A. Svoboda \\ University of Nebraska Medical Center, Omaha \\ Ronald Cerny \\ University of Nebraska - Lincoln, rcerny1@unl.edu \\ Keith R. Johnson \\ University of Nebraska Medical Center, Omaha, kjohnsonr@unmc.edu \\ Margaret J. Wheelock \\ University of Nebraska Medical Center, Omaha, mwheelock@unmc.edu
}

Follow this and additional works at: https://digitalcommons.unl.edu/chemistrycerny

Part of the Chemistry Commons

Fukunaga, Yoshitaka; Svoboda, Robert A.; Cerny, Ronald; Johnson, Keith R.; and Wheelock, Margaret J., "Expression artifact with retroviral vectors based on pBMN" (2009). Ronald Cerny Publications. 9. https://digitalcommons.unl.edu/chemistrycerny/9

This Article is brought to you for free and open access by the Published Research - Department of Chemistry at DigitalCommons@University of Nebraska - Lincoln. It has been accepted for inclusion in Ronald Cerny Publications by an authorized administrator of DigitalCommons@University of Nebraska - Lincoln. 


\title{
Expression artifact with retroviral vectors based on $\mathrm{pBMN}$
}

\author{
Yoshitaka Fukunaga, ${ }^{1}$ Robert A. Svoboda, ${ }^{1}$ Ronald L. Cerny, ${ }^{2}$ \\ Keith R. Johnson, ${ }^{1,3,4}$ and Margaret J. Wheelock ${ }^{1,3,4}$ \\ 1. Department of Oral Biology, University of Nebraska Medical Center, Omaha, NE 68198, USA \\ 2. Department of Chemistry, University of Nebraska-Lincoln, Lincoln, NE 68588, USA \\ 3. Eppley Institute for Research in Cancer and Allied Diseases, University of Nebraska Medical Center, Omaha, NE 68198, USA \\ 4. Eppley Cancer Center, University of Nebraska Medical Center, Omaha, NE 68198, USA \\ Corresponding author - K. R. Johnson, Department of Oral Biology, University of Nebraska Medical Center, \\ Omaha, NE 68198, USA; fax 402 559-3888; email kjohnsonr@unmc.edu
}

\begin{abstract}
While characterizing various splice forms of p120 catenin, we observed what appeared to be a novel posttranslational modification of p120, resulting in a higher molecular weight form that was dependent on the splicing pattern. Further investigation revealed the higher molecular weight form to be a fusion protein between sequences encoded by the retroviral vector and p120. We found that the publicly available sequence of the vector we used does not agree with the experimental sequence. We caution other investigators to be aware of this potential artifact.

Keywords: p120 Catenin, pBMN, LZRS, Retroviral vector

Abbreviations: DMEM, Dulbecco's modified Eagle's medium; mAb, monoclonal antibody; HA, hemagglutinin; MoMLV, Moloney murine leukemia virus; TBS, Tris-buffered saline; cDNA, complementary DNA; PBS, phosphate-buffered saline; EDTA, ethylenediaminetetraacetic acid; PMSF, phenylmethylsulfonyl fluoride; SDS, sodium dodecyl sulfate; PAGE, polyacrylamide gel electrophoresis; MS, mass spectrometry; IgG, immunoglobulin G
\end{abstract}

The cytoplasmic tails of classical cadherins directly bind both $\beta$-catenin and p120 catenin [1]. In contrast to $\beta$-catenin, p120 [2] undergoes extensive alternative splicing leading to the expression of multiple protein isoforms [3]. There are four possible start codons, with proteins initiating at the most upstream start codon termed isoform 1 . In addition, there are three exons (termed A, B, and C) that are included variably. Thus, the longest possible protein would be termed p120-1ABC.

As part of an ongoing project, we obtained constructs encoding isoforms p120-1A and p120-1AC [4]. When we expressed these constructs in cells using a retroviral vector based on LZRS [5], we observed a modification of p120 that appeared to be exon $\mathrm{C}$ dependent. However, further analysis showed that this was an expression vector artifact, and these data are presented below.

\section{Materials and methods}

\section{Cell culture}

A431 cells (American Type Culture Collection, Manassas, VA, USA) and S2-013 cells [6] were grown in Dulbecco's modified Eagle's medium (DMEM) supplemented with $10 \%$ fetal calf serum at $37^{\circ} \mathrm{C}$ in a $5 \% \mathrm{CO}_{2}$ humidified atmosphere.

\section{Reagents and sources of antibodies}

Reagents were obtained from Sigma-Aldrich (St. Louis, MO, USA) or Fisher Chemicals (Fairlawn, NJ, USA) unless otherwise indicated. Anti-p120 mouse monoclonal antibody
(mAb, pp120) was purchased from BD Biosciences (Franklin Lakes, NJ, USA). Anti-hemagglutinin (HA) mouse mAb (H3663) was obtained from Sigma-Aldrich. Anti- $\beta$-tubulin mouse mAb (E7) was obtained from the Developmental Studies Hybridoma Bank (Iowa City, IA, USA). Rabbit polyclonal antiserum against Moloney murine leukemia virus (MoMLV) integrase was kindly provided by Monica J. Roth [7]. The antiserum against the integrase was diluted 1:1000 in Tris-buffered saline (TBS: $10 \mathrm{mM}$ Tris- $\mathrm{HCl}[\mathrm{pH} 8.0]$ and $150 \mathrm{mM} \mathrm{NaCl}$ ) for immunoblotting. The MoMLV gag p15 mouse mAb (hybridoma supernatant from clone 34) was a kind gift from Bruce Chesebro [8].

\section{cDNA constructs, transfections, and infections}

Full-length, HA-tagged human p120 complementary DNAs (cDNAs) were kind gifts from Xiang-Jiao Yang (McGill University, Montreal, Canada). p120-1AC contains an extra six amino acids encoded by exon $\mathrm{C}$ in addition to p120-1A as described previously (see Figure 1A) [4]. The p120-1A and p120$1 \mathrm{AC}$ cDNAs were inserted into a derivative of the LZRS-neo retroviral vector [9]. (Details of the construction are available on request.) LZRS was derived from vectors based on pBMN [5]. Constructs were transfected into Phoenix packaging cells using TransIT-LT1 Reagent (Mirus, Madison, WI, USA). Conditioned medium containing recombinant retrovirus was supplemented with $4 \mu \mathrm{g} / \mathrm{ml}$ polybrene and added to target cells as described previously [6]. Transfected Phoenix cells were selected with $2 \mu \mathrm{g} / \mathrm{ml}$ puromycin, and infected target cells were selected with $1 \mathrm{mg} / \mathrm{ml} \mathrm{G} 418$. 

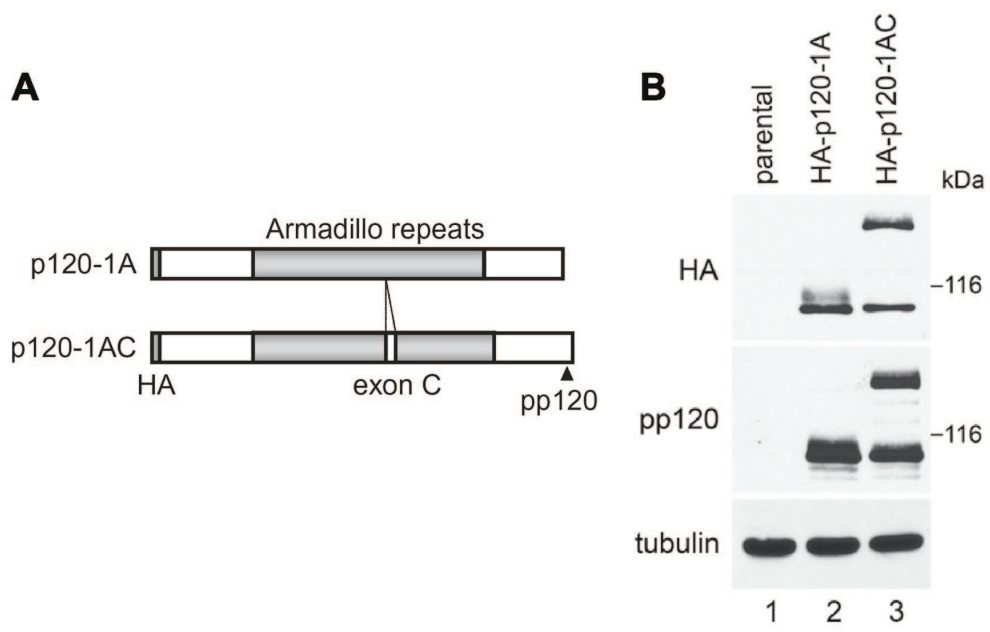

Figure 1. (A) The structures of the p120-1A and p120-1AC constructs are shown. In addition to p120-1A, p120-1AC contains an extra six amino acids encoded by exon $\mathrm{C}$ in the middle of the Armadillo repeats. The N-terminal HA epitope and the epitope recognized by pp120 are shown. (B) Extracts of parental S2-013 cells and cells infected with the p120-1A and p120-1AC constructs were prepared. Here $40 \mu \mathrm{g}$ was separated by SDSPAGE and immunoblotted with either anti-HA or pp120, an anti-p120 mAb that recognizes an epitope near the C terminus of p120. A band of approximately $110 \mathrm{kDa}$ is seen in both lanes 2 and 3. A band of approximately $135 \mathrm{kDa}$ appears only in the extract of cells expressing the p120-1AC construct. The slightly larger bands seen most prominently in lane 2 probably represent phospho-variants of p120 [6]. $\beta$-Tubulin was used as a loading control. The position of the 116-kDa molecular weight marker is indicated.

\section{Extraction of cells, purification of proteins, MS, and N-termi- nal sequencing}

Confluent monolayers of cells were rinsed three times with phosphate-buffered saline (PBS) and extracted on ice with TNE buffer (10 mM Tris-HCl [pH 8.0], 0.5\% Nonidet P-40, and $1 \mathrm{mM}$ ethylenediaminetetraacetic acid [EDTA]) containing $1 \mathrm{mM}$ phenylmethylsulfonyl fluoride (PMSF). Extracts were centrifuged at $14,000 \mathrm{rpm}$ for $15 \mathrm{~min}$ at $4{ }^{\circ} \mathrm{C}$, and the supernatant was collected. Protein concentration was determined using a Bio-Rad protein assay kit (Hercules, CA, USA).

The protein was purified by immunoprecipitation using an anti-HA antibody agarose conjugate (Sigma-Aldrich) according to the manufacturer's protocol. In brief, cells were extracted on ice with RIPA buffer (50 mM Tris- $\mathrm{HCl}$ [pH 8.0], $1 \%$ Nonidet P-40, $0.5 \%$ sodium deoxycholate, $0.1 \%$ sodium dodecyl sulfate [SDS], and $150 \mathrm{mM} \mathrm{NaCl}$ ) containing protease inhibitor cocktail (Calbiochem, La Jolla, CA, USA). The cell extracts were incubated with anti-HA agarose conjugate overnight at $4{ }^{\circ} \mathrm{C}$, and the agarose beads were washed five times with RIPA buffer. The immunoprecipitated HA-tagged proteins were eluted with $200 \mu \mathrm{g} / \mathrm{ml}$ HA peptide (cat. no. I2149, Sigma-Aldrich) for $1 \mathrm{~h}$ or with SDS sample buffer. The supernatant was collected, resolved by SDS-polyacrylamide gel electrophoresis (PAGE), and transferred to nitrocellulose for mass spectrometry (MS) or PVDF membranes for Nterminal sequencing. The MS data were analyzed using the Mascot search engine. The samples for sequencing were sent to the Protein Structure Core Facility at the University of Nebraska Medical Center.

\section{Immunoprecipitation and immunoblotting}

Proteins $(2.0 \mathrm{mg})$ were added to $300 \mu \mathrm{l}$ of hybridoma-conditioned medium and gently mixed at $4{ }^{\circ} \mathrm{C}$ for $2 \mathrm{~h}$. Then $50 \mu \mathrm{l}$ of packed anti-mouse immunoglobulin G (IgG) affinity gel (MP Biomedicals, Solon, OH, USA) was added, and mixing continued overnight. Immune complexes were washed five times with TBST buffer (10 mM Tris- $\mathrm{HCl}$ [pH 8.0], $150 \mathrm{mM} \mathrm{NaCl}$, and $0.05 \%$ Tween 20). After the final wash, the packed beads were resuspended in $50 \mu \mathrm{l}$ of $2 \times$ Laemmli sample buffer and boiled for $5 \mathrm{~min}$, and then the proteins were resolved by SDSPAGE. Proteins were electrophoretically transferred overnight to nitrocellulose membranes and immunoblotted as described previously [10].

\section{Sequencing a portion of $p B M N-Z$ and LZRS}

The amino acid sequence in Table $2 \mathrm{~B}$ was deduced from the nucleotide sequence of LZRS obtained with the primers CTCCGTCTGAATTTTTGC and GGTCAAGCCCTTTGTACAC that prime upstream of the gag start codon and in the p15 open reading frame, respectively. $\mathrm{pBMN}-\mathrm{Z}$ (purchased from Addgene, Cambridge, MA, USA) was sequenced with the same primers and found to contain sequence identical to LZRS.

\section{Results and discussion}

Constructs encoding isoforms p120-1A and p120-1AC that were N-terminally HA-tagged (YPYDVPDYA) [4] were ligated into the retroviral expression vector LZRS. The constructs were transfected into Phoenix packaging cells [5], and the retroviral particles were used to infect target cells. Normally, p120-1A would be expected to migrate at approximately $110 \mathrm{kDa}$, and this was observed (Figure 1B). Because exon $\mathrm{C}$ is only $18 \mathrm{nt}$ (Figure 1A) [3], p120-1AC is also expected to migrate at approximately $110 \mathrm{kDa}$. However, when p120$1 \mathrm{AC}$ was expressed in cells, we observed two bands positive with both anti-HA and the mAb pp120 that recognize an epitope near the $C$ terminus of p120 [11]. One of these two bands essentially comigrated with isoform p120-1A at $110 \mathrm{kDa}$, whereas another band of approximately equal intensity was observed at approximately $135 \mathrm{kDa}$ (Figure 1B). We observed the same two bands when p120-1AC was expressed in S2-013, A431, HeLa, MiaPaCaII, MDA-MB-468, SCC-1, MCF7, and Phoenix packaging cells.

Initial experiments showed that the 135-kDa band was not identified by anti-ubiquitin antibodies or antibodies against the 
Table 1. Experimental and predicted amino acid sequences.

\begin{tabular}{|c|c|c|c|c|c|c|c|c|c|c|}
\hline Deduced sequence of cDNA & M & $\mathrm{Y}$ & $\mathrm{P}$ & $\mathrm{Y}$ & $\mathrm{D}$ & $\mathrm{V}$ & $\mathrm{P}$ & D & Y & A \\
\hline $\mathrm{N}$ terminus of $110-\mathrm{kDa}$ band & M & $\mathrm{Y}$ & $\mathrm{P}$ & $\mathrm{Y}$ & $\mathrm{D}$ & V & $\mathrm{P}$ & $\mathrm{D}$ & Y & A \\
\hline $\mathrm{N}$ terminus of $135-\mathrm{kDa}$ band & $\mathrm{P}$ & (L) & & $\mathrm{L} / \mathrm{Q}$ & $\mathrm{T}$ & V & (V) & $\mathrm{T}$ & $\mathrm{P}$ & (L) \\
\hline Deduced sequence of LZRS gaga & G & $\mathrm{P}$ & G & $\mathrm{Q}$ & $\mathrm{T}$ & V & $\mathrm{T}$ & $\mathrm{T}$ & $\mathrm{P}$ & $\mathrm{L}$ \\
\hline
\end{tabular}

Positions that were ambiguous are in parentheses.

a. The gag sequence presented in the table starts with amino acid 2 in the open reading frame.

SUMO family members (results not shown). The lack of processing intermediates between the 135- and 110-kDa bands, as well as the intensity of the 135-kDa band, suggested a novel posttranslational modification of p120 that was exon $\mathrm{C}$ dependent.

Using an anti-HA resin, we purified the 110- and 135-kDa proteins from extracts of A431 and S2-013 cells that had been infected with the p120-1AC retroviral construct. Bands cut from Coomassie blue-stained gels were digested with trypsin, the peptides were processed for MS, and the results were searched against the human database. In some experiments, we obtained tryptic peptides that represented up to $70 \%$ coverage of the p120-1AC open reading frame. However, even after multiple MS experiments, we failed to identify candidates that could have caused the shift in apparent molecular weight.

Table 2. Amino acid sequences of the MoMLV virus polyprotein and a portion of the LZRS vector.

(A) The sequence of the MoMLV polyprotein
1 MGQTVTTPLS LTLDHWKDVE
51 PRDGTFNRDL ITQVKIKVFS PGPHGHPDQV PYIVTWEALA FDPPPWAKPF
101 VHPKPPPPLP PSAPSLPLEP PLSTPSRSSL YPALTPSLGA KPKPQVLSDS
151 EGPLIDLLTE DPPPYRDPRP PPSDGDGNSG EATPAGEAPD PSPMASRLRG
201 RREPPVADST TSQAFPLRTG GNGQLQYWPF SSSDLYNWKN NNPSFSEDPG
251 KLTALIESVL ITHQPTWDDC QQLLGTLLTG EEKQRVLLEA RKAVRGDDGR
301 PTQLPNEVDA AFPLERPDWE YTTQAGRNHL VQYRQLLLAG LQNAGRSPTN
351 LAKVKGITQG PNESPSAFLE RLKEAYRRYT PYDPEDPGQE TNVAMSFIWQ
401 SAPDIGRKLE RLEDLKNKTL GDLVREAERI FNKRETPEER EERIRRETEE
451 KEERRRTEDE QKEKERDRRR HREMSKLLAT VVSGQRQDRQ GGERRRSQLD
501 RDQCAYCKEK GHWAKDCPKK PRGPRGPRPQ TSLLTLDDOG GOGOEPPPEP
551 RITLKVGGOP VTFLVDTGAO HSVLTONPGS LSGKSAWVOG ATGGKRYRWT
601 TDRKVHLATG KVTHSFLHVP DCPYPLLGRD LLTKLKAOIH FEGSGAOVMG
651 PMGQPLOVLT LNIEDEYRLH ETSKEPDVSL GSTWLSDFPQ AWAETGGMGL
701 AVRQAPLIIP LKATSTPVSI KQYPMSQEAR LGIKPHIQRL LDQGILVPCQ
751 SPWNTPLLPV KKPGTNDYRP VQDLREVNKR VEDIHPTVPN PYNLLSGLPP
801 SHQWYTVLDL KDAFFCLRLH PTSQPLFAFE WRDPEMGISG QLTWTRLPQG
851 FKNSPTLFDE ALHRDLADFR IQHPDLILLQ YVDDLLLAAT SELDCQQGTR
901 ALLQTLGDLG YRASAKKAQI CQKQVKYLGY LLKEGQRWLT EARKETVMGQ
951 PTPKTPRQLR EFLGTAGFCR LWIPGFAEMA APLYPLTKTG TLFDWGPDQQ
1001 KAYQEIKQAL LTAPALGLPD LTKPFELFVD EKQGYAKGVL TQKLGPWRRP
1051 VAYLSKKLDP VAAGWPPCLR MVAAIAVLTK DAGKLTMGQP LVILAPHAVE
1101 ALVKQPPDRW LSNARMTHYQ ALLLDTDRVQ FGPVVALNPA TLLPLPEEGL
1151 QHNCLDILAE AHGTRPDLTD QPLPDADHTW YTDGSSFLQE GQRKAGAAVT
1201 TETEVIWAKA LPAGTSAQRA ELIALTQALK MAEGKKLNVY TDSRYAFATA
1251 HIHGEIYRRR GLLTSEGKEI KNKGEILALL KALFLPKRLS IIHCPGHQKG
1301 HSAEARGNRM ADQAARKAAI TETPDTSTLL IEKSSPYTSE HFHYTVTDIK
1351 DLTKLGAIYD KTKKYWVYOG KPVMPDOFTF ELLDFLHOLT HLSFSKTKAL
1401 LERSHSPYYM LNRDRTLKNI TETCKACAOV NASKSAVKOG TRVRGHRPGT
1451 YWEIDFTEIK PGLYGYKYLL VFIDTFSGWI EAFPTKKETA KVVTKKLLEE
1501 IFPRFGMPOV LGTDNGPAFV SKVSOTVADL LGIDWKLHCA YRPOSSGOVE
1551 RMNRTIKETL TKLTLATGSR DWVLLLPLAL YRARNTPGPH GLTPYEILYG
1601 APPPLVNFPD PDMTRVTNSP SLOAHLQALY LVOHEVWRPL AAAYOEOLDR
1651 PVVPHPYRVG DTVWVRRHOT KNLEPRWKGP YTVLLTTPTA LKVDGIAAWI
1701 HAAHVKAADP GGGPSSRLTW RVORSONPLK IRLTREAP

(B) The deduced amino acid sequence of the LZRS vector

1 MGPGQTVTTP LSLTLDHWKD VERIAHNQSV DVKKRRWVTF CSAEWPTFNV

51 GWPRDGTFNR DLITQVKIKV FSPGPHGHPD QVPYIVTWEA LAFDPPPWAK 101 PFVHPKPPPP LPPSAPSLPL EPPLSTPSRS SLYPALTPSL GAPIWGLTPY 151 EILYGAPPPL VNFPDPDMTR VTNSPSLQAH LQALYLVQHE VWRPLAAAYQ 201 EQLDRPVVPH PYRVGDTVWV RRHQTKNLEP RWKGPYTVLL TTPTALKVDG 251 IAAWIHAAHV KAADPGGGPS SRL

\section{gag}

protease

polymerase

\author{
integrase
}

Note for A. The sequence of the MoMLV gag-pro-pol-int polyprotein is shown with the gag, protease, polymerase, and integrase proteins italicized or underlined (GenBank AAL69910). Peptides identified by MS are shown in red.

Note for B. The deduced amino acid sequence of a portion of the LZRS vector encoding a 273-amino-acid polypeptide is shown. Peptides identified by MS are shown in red. The deduced sequence differs from AAL69910 by the insertion of two residues near the N terminus (PG, underlined) and three residues encoded by a linker (PIW, underlined) joining the gag and integrase sequences. The p15 gag protein ends with Y133. Pro3 in the LZRS-encoded sequence likely interferes with the myristoylation of gag [14]. 


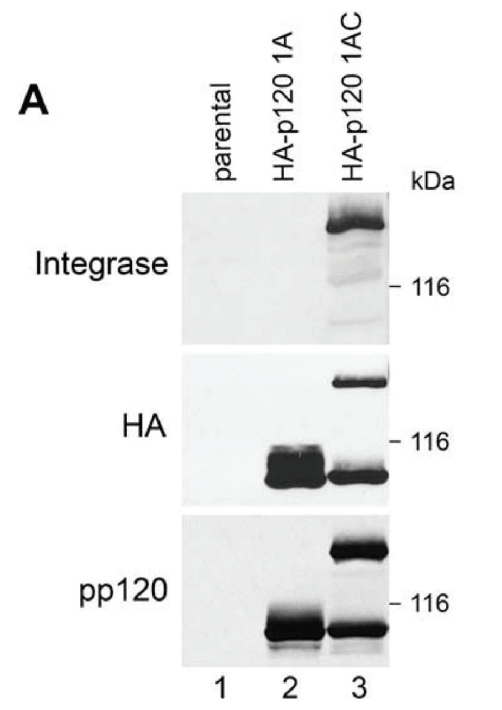

B

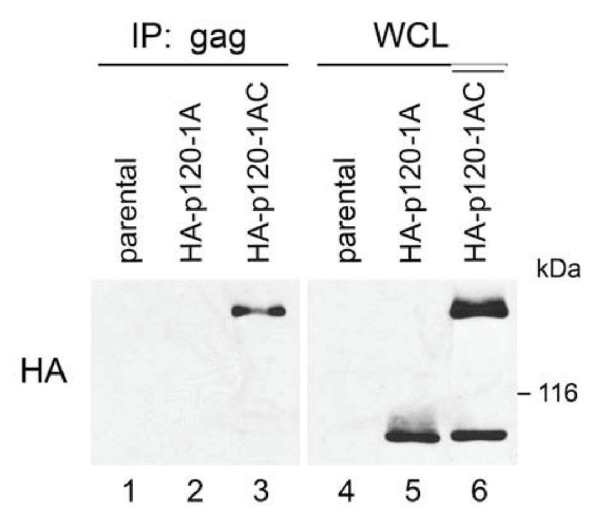

Figure 2. (A) Extracts were prepared and immunoblotted as described in Figure 1. The 135-kDa band (lane 3), but not the 110-kDa band, was identified by the anti-integrase antiserum. (B) Cell extracts were immunoprecipitated using a mAb that recognizes MoMLV p15 (gag), and the immunoprecipitates (IP) were immunoblotted with anti-HA. For comparison, whole cell lysates (WCL) blotted with anti-HA are shown. The 135-kDa band (lane 3), but not the 110-kDa band, was immunoprecipitated by the anti-p15 antibody. The position of the 116-kDa molecular weight marker is indicated.

We then sent the 110- and 135-kDa bands for N-terminal sequence analysis. The N-terminal sequences of the 110 - and 135-kDa proteins are shown in Table 1 along with the deduced amino acid sequence based on the experimentally determined nucleotide sequence of the p120-1AC construct. The N-terminal sequence of the $110-\mathrm{kDa}$ band clearly matched the expected sequence of methionine followed by the HA epitope. However, the N-terminal sequence of the $135-\mathrm{kDa}$ band, although somewhat ambiguous, was clearly different from that of the 110-kDa band with no evidence of an HA epitope.

The N-terminal sequencing data together with the immunoblotting data suggested that the $135-\mathrm{kDa}$ protein consisted of the full-length, HA-tagged p120-1AC construct fused at its $\mathrm{N}$ terminus to an unidentified protein. However, the MS experiments failed to identify this fusion partner in the human database.

The LZRS retroviral vector system was constructed using MoMLV [5]. Cells infected with MoMLV express several proteins, including the gag-pro-pol-int (gag, protease, polymerase, and integrase) fusion protein (GenBank AAL69910) shown in Table 2A [12]. When the tryptic peptides obtained from the $135-\mathrm{kDa}$ band were searched against the viral database, several peptides from the gag-pro-pol-int protein of MoMLV (highlighted in red in Table 2A) were identified.

These data prompted us to determine the nucleotide sequence of the relevant portion of the LZRS vector (Table 2B). We found that the LZRS vector contains a 273-amino-acid open reading frame beginning with the methionine codon of the MoMLV gag gene and continuing on through all of the viral sequences that are upstream of the multiple cloning site of the expression vector. The deduced 273-amino-acid protein contains the entire retroviral p15 protein that is derived from the gag protein (with 2 amino acids inserted near the $\mathrm{N}$ terminus compared with AAL69910), a small portion of the retroviral p12 protein also derived from gag, a 3-amino-acid sequence encoded by a linker, and 128 amino acids of the viral integrase protein. Although not an exact match, the N-terminal sequence of the deduced protein is similar to the N-terminal amino acid sequence obtained experimentally from the
135-kDa band (Table 1). As fortune would have it, if we continued the conceptual translation of the p120-1AC construct downstream of the viral sequences into the multiple cloning site, the gag-int protein shown in Table 2A was in-frame with the HA tag and the p120 cDNA. However, due to differences in the 5 '-end sequences between the p120-1A and p120-1AC constructs, the gag-int protein was not in-frame with HA or p120 in the p120-1A construct.

To determine whether the $135-\mathrm{kDa}$ band was indeed a fusion between portions of the MoMLV gag-pro-pol-int protein with p120, we obtained antibodies against both the MoMLV integrase [13] and MoMLV p15 (mAb 34) [8] proteins. As shown in Figure 2A, the anti-integrase antiserum identified the $135-\mathrm{kDa}$ protein, but not the $110-\mathrm{kDa}$ protein, in immunoblots. Although in our hands the $\mathrm{mAb}$ recognizing p15 did not work in immunoblots, this antibody immunoprecipitated the 135-kDa band, but not the 110-kDa band, from cell extracts (Figure 2B). These data show that the $135-\mathrm{kDa}$ protein is a fusion between sequences encoded by the expression vector and by the HA-tagged p120 cDNA.

LZRS contains both the MoMLV splice donor site, located approximately 415 bases upstream of the gag AUG, and the splice acceptor site, located in the integrase-encoding segment [12]. If transcripts produced from LZRS are spliced using these donor and acceptor sites, the gag AUG along with all of the p15 coding region, as well as much of the integrase coding region, should be spliced out. However, our data suggest that splicing is not complete because the $135-\mathrm{kDa}$ fusion protein is as abundant as the normal-sized p120 (Figure 2).

We previously used LZRS vectors without encountering this artifact. It is interesting to note that with the integrase antiserum we also observed a 30-kDa band in cells infected with the empty LZRS vector, implying that the gag-int protein is typically produced (results not shown).

The publicly available sequence of $\mathrm{pBMN}-\mathrm{Z}$ and related vectors (see http://www.addgene.org and http://0-www. stanford.edu.library.unl.edu/group/nolan/plasmid_maps/ pmaps.html) do not agree with our sequence of LZRS. These sequences show no open reading frame across the MoMLV se- 
quences. However, when we purchased pBMN-Z from Addgene and sequenced the relevant region, we found that indeed pBMN-Z contains the same open reading frame that we found in LZRS due to the presence of two nucleotide differences in the publicly available pBMN sequence. The 5' LTR and $\Psi$ packaging sequences in $\mathrm{pBMN}$ were derived from the vector MFG [5]. The sequence of MFG (US patent 6,544,771) agrees with the sequence we found in LZRS and not that of the publicly available sequence of $\mathrm{pBMN}$. Thus, it is likely that all vectors based on the pBMN series, such as LZRS, have the potential to express artifactual fusion proteins.

To prevent the artifact we saw with the p120-1AC construct from occurring, we recommend that investigators place a stop codon upstream of the desired start codon, either in the gag reading frame or in the reading frame of their constructs. We have done this with the p120-1AC construct and no longer see the $135-\mathrm{kDa}$ band (results not shown).

\section{Acknowledgments}

We thank Laurey Steinke of the Protein Structure Core Facility in the Department of Biochemistry and Molecular Biology at the University of Nebraska Medical Center for determining the N-terminal sequences and for engaging in helpful discussions. We also thank Xiang-Jiao Yang for constructs and thank Monica J. Roth and Bruce Chesebro for antibodies. The work was supported by Grant GM51188 from the N.I.H. to M.J.W. and Grants DE12308 and CA137401 from the N.H. to K.R.J.

\section{References}

[1] M. J. Wheelock and K. R. Johnson, Cadherins as modulators of cellular phenotype, Annu. Rev. Cell Dev. Biol. 19 (2003), pp. 207-235.

[2] A. B. Reynolds, P120-Catenin: past and present, Biochim. Biophys. Acta 1773 (2007), pp. 2-7.

[3] A. Keirsebilck, S. Bonne, K. Staes, J. van Hengel, F. Nollet, A. Reynolds, and F. van Roy, Molecular cloning of the human p120ctn catenin gene (CTNND1): expression of multiple alternatively spliced isoforms, Genomics 50 (1998), pp. 129-146.

[4] S. C. Kim, R. Sprung, Y. Chen, Y. Xu, H. Ball, J. Pei, T. Cheng, Y. Kho, H. Xiao, L. Xiao, N. V. Grishin, M. White, X. J. Yang, and Y.
Zhao, Substrate and functional diversity of lysine acetylation revealed by a proteomics survey, Mol. Cell 23 (2006), pp. 607-618.

[5] T. M. Kinsella and G. P. Nolan, Episomal vectors rapidly and stably produce high-titer recombinant retrovirus, Hum. Gene Ther. 7 (1996), pp. 1405-1413.

[6] Y. Fukumoto, Y. Shintani, A. B. Reynolds, K. R. Johnson, and M. J. Wheelock, The regulatory or phosphorylation domain of p120 catenin controls E-cadherin dynamics at the plasma membrane, Exp. Cell Res. 314 (2008), pp. 52-67.

[7] K. Bupp, A. Sarangi, and M. J. Roth, Selection of feline leukemia virus envelope proteins from a library by functional association with a murine leukemia virus envelope, Virology 351 (2006), pp. 340-348.

[8] B. Chesebro, W. Britt, L. Evans, K. Wehrly, J. Nishio, and M. Cloyd, Characterization of monoclonal antibodies reactive with murine leukemia viruses: Use in analysis of strains of Friend MCF and Friend ecotropic murine leukemia virus, Virology 127 (1983), pp. 134-148.

[9] R. C. Ireton, M. A. Davis, J. van Hengel, D. J. Mariner, K. Barnes, M. A. Thoreson, P. Z. Anastasiadis, L. Matrisian, L. M. Bundy, L. Sealy, B. Gilbert, F. van Roy, and A. B. Reynolds, A novel role for p120 catenin in E-cadherin function, J. Cell Biol. 159 (2002), pp. $465-476$.

[10] J. K. Wahl III, Y. J. Kim, J. M. Cullen, K. R. Johnson, and M. J. Wheelock, N-Cadherin-catenin complexes form prior to cleavage of the proregion and transport to the plasma membrane, J. Biol. Chem. 278 (2003), pp. 17269-17276.

[11] X. Xia, R. H. Carnahan, M. H. Vaughan, G. A. Wildenberg, and A. B. Reynolds, P120 serine and threonine phosphorylation is controlled by multiple ligand-receptor pathways but not cadherin ligation, Exp. Cell Res. 312 (2006), pp. 3336-3348.

[12] T. M. Shinnick, R. A. Lerner, and J. G. Sutcliffe, Nucleotide sequence of Moloney murine leukaemia virus, Nature 293 (1981), pp. 543-548.

[13] J. Puglia, T. Wang, C. Smith-Snyder, M. Cote, M. Scher, J. N. Pelletier, S. John, C. B. Jonsson, and M. J. Roth, Revealing domain structure through linker-scanning analysis of the murine leukemia virus (MuLV) RNase $\mathrm{H}$ and MuLV and human immunodeficiency virus type 1 integrase proteins, J. Virol. 80 (2006), pp. 9497-9510.

[14] T. Utsumi, M. Sato, K. Nakano, D. Takemura, H. Iwata, and R. Ishisaka, Amino acid residue penultimate to the amino-terminal Gly residue strongly affects two cotranslational protein modifications, $N$-myristoylation and N-acetylation, J. Biol. Chem. 276 (2001), pp. 10505-10513. 